\title{
CONVOLUTION, FOURIER TRANSFORM AND SOBOLEV SPACES GENERATED BY NON-LOCAL IONKIN PROBLEM
}

\author{
B.E. KANGUZHIN, N.E. TOKMAGAMBETOV
}

\begin{abstract}
In this work, given a second order differential operator $\mathcal{B}$ subject to non-local boundary conditions, we assign Fourier transform and convolution to this problem. We study the properties of the introduced convolution and describe the class of test functions. We also introduce Sobolev spaces and obtain Plancherel identity related to operator $\mathcal{B}$.
\end{abstract}

Keywords. convolution, Fourier transform, nonlocal boundary condition, test functions, Sobolev space, Plancherel identity, differential operator, Ionkin problem.

Mathematics Subject Classification: 43A99, 46F12, 42A16, 34B10, 45J05

\section{INTRODUCTION}

The standard Fourier transform is a unitary transform in Hilbert space $L_{2}(0, b)$ and it is generated by the differentiation operator $\left(-i \frac{d}{d x}\right)$ since the system of exponentials $\{\exp (i \lambda x), \lambda \in$ $\mathbb{R}\}$ is a system "eigenfunctions" associated with its continuous spectrum. Bilinear commutative associative convolution without annihilators is very close related with Fourier transform. In papers [1]-44, instead of the differentiation operator $\left(-i \frac{d}{d x}\right)$ in space $L_{2}(-\infty, \infty)$, there was considered the operator generated by the differential expression $\left(-i \frac{d}{d x}\right)$ in Hilbert space $L_{2}(0, b)$ as $b<\infty$ and there were introduced Fourier transform and the convolution generated by this operator. In paper [5], a Fourier analysis generated by a differential operator in a bounded domain with simple eigenvalues was developed. The main difference of the present paper from paper [5] is that the generating operator has multiple eigenvalues. In the paper we introduce the notion of Fourier transform and convolution generated by the operator of double differentiation in space $L_{2}(0,1)$ subject to nonlocal boundary conditions studied in work [6]. It is known what role is played by generalized solutions to differential equations. Nonlocal boundary value operators happen to produce its own class of test functions. This is why new classes of generalized functions appear which reflect features of nonlocal boundary conditions.

In Hilbert space $L_{2}(0,1)$ we define operator $\mathcal{B}$ generated by the differential expression

$$
l(u) \equiv-\frac{d^{2} u(x)}{d x^{2}}, \quad 0<x<1,
$$

on the domain

$$
D(\mathcal{B})=\left\{u \in W_{2}^{2}[0,1]: u(0)=0, u^{\prime}(0)=u^{\prime}(1)\right\} .
$$

Its spectral properties were studied in details in work by N.I. Ionkin [6]. Operator $\mathcal{B}$ is not self-adjoint, but the system of its eigenfunctions and adjoint functions is a basis in $L_{2}(0,1)$. It gives an opportunity to develop a nonharmonic analysis related with operator $\mathcal{B}$. A nonharmonic

B.E. Kanguzhin, N.E. Tokmagambetov, Convolution, Fourier transform and Sobolev spaces GENERATED BY NON-LOCAL IONKIN PROBLEM.

(C) Kanguzhin B.E., Tokmagambetov N.E. 2015.

The first author was supported by the Committee of Science of the Ministery of Education and Science of the Republic of Kazakhstan (grant no. 0757/GF4). The second author is supported by the Committee of Science of the Ministery of Education and Science of the Republic of Kazakhstan (grant no. 0773/GF4).

Submitted February 19, 2015. 
analysis generated by the exponential systems was studied in details in works by A.M. Sedletskii [7], [8. In further works we shall introduce pseudodifferential operators and other elements of the harmonic analysis generated by operator $\mathcal{B}$.

We denote by $\mathcal{B}^{*}$ the adjoint operator for $\mathcal{B}$; this operator is generated by differential expression (1.1) and the boundary conditions

$$
v^{\prime}(1)=0, \quad v(0)=v(1)
$$

Operator $\mathcal{B}$ has the eigenvalues

$$
\lambda_{k}=(2 \pi k)^{2}, \quad k=0,1, \ldots,
$$

and the eigenfunctions and the adjoint functions read as

$$
u_{0}(x)=x, \quad u_{2 k-1}(x)=\sin (2 \pi k x), \quad u_{2 k}(x)=x \cos (2 \pi k x), \quad k=1,2, \ldots
$$

It means that as $k>0$, functions in $(1.3)$ belong to $D(\mathcal{B})$ and solve the differential equations

$$
-u_{2 k-1}^{\prime \prime}(x)=\lambda_{k} u_{2 k-1}(x), \quad-u_{2 k}^{\prime \prime}(x)=\lambda_{k} u_{2 k}(x)+2 \sqrt{\lambda_{k}} u_{2 k-1}(x) .
$$

Eigenfunction $u_{2 k-1}(x)$ and adjoint function $u_{2 k}(x)$ are associated with each eigenvalue $\lambda_{k}$ for $k>0$.

The eigenvalues of operator $\mathcal{B}^{*}$ are

$$
\mu_{k}=(2 \pi k)^{2}, \quad k=0,1, \ldots,
$$

and eigenfunctions and adjoint functions are

$$
v_{0}(x)=2, \quad v_{2 k-1}(x)=4(1-x) \sin (2 \pi k x), \quad v_{2 k}(x)=4 \cos (2 \pi k x), \quad k=1,2, \ldots
$$

At that, eigenfunction $v_{2 k}(x)$ and adjoint function $v_{2 k-1}(x)$ are associated with eigenvalue $\mu_{k}$ for $k>0$, i.e., functions in (1.4) satisfy the relations

$$
-v_{2 k}^{\prime \prime}(x)=\mu_{k} v_{2 k}(x), \quad-v_{2 k-1}^{\prime \prime}(x)=\mu_{k} v_{2 k-1}(x)+2 \sqrt{\mu_{k}} v_{2 k}(x)
$$

and boundary conditions 1.2 .

We cite the following results of work [6].

Lemma 1.1. Sequences of functions (1.3) and (1.4) form a biorthogonal system of functions on the interval $(0,1)$ such that for each $i, j \in \mathbb{N}$ the relation

$$
\left(u_{2 i-k}, v_{2 j-l}\right)=\int_{0}^{1} u_{2 i-k}(x) v_{2 j-l}(x) d x=\delta_{2 i-k, 2 j-l}, \quad k=0, m_{i}-1 ; \quad l=0, m_{j}-1,
$$

holds true. Here $\delta_{2 i-k, 2 j-l}$ is the Kronecker delta, $m_{0}=1$, and $m_{i}=2, i \geqslant 1$.

Theorem 1.1. The sequence of functions

$$
u_{0}(x)=x, \quad u_{2 k-1}(x)=\sin (2 \pi k x), \quad u_{2 k}(x)=x \cos (2 \pi k x), \quad k \in \mathbb{N}
$$

forms a Riesz basis in space $L_{2}(0,1)$.

\section{Convolution Generated By Operator $\mathcal{B}$}

In space $L_{2}(0,1)$ we introduce the convolution by the formula

$$
\begin{aligned}
(g * f)(x)= & \frac{1}{2} \int_{x}^{1} g(1+x-t) f(t) d t+\frac{1}{2} \int_{1-x}^{1} g(x-1+t) f(t) d t \\
& +\int_{0}^{x} g(x-t) f(t) d t-\frac{1}{2} \int_{0}^{1-x} g(1-x-t) f(t) d t+\frac{1}{2} \int_{0}^{x} g(1+t-x) f(t) d t .
\end{aligned}
$$

Lemma 2.1. a) The introduced convolution is bilinear, commutative and associative for each $f, g \in L_{2}(0,1)$. 
b) The resolvent of operator $\mathcal{B}$ has the convolution representation

$$
(\mathcal{B}-\lambda I)^{-1} f=g * f,
$$

where $g(x)=\frac{\sin \sqrt{\lambda} x}{\sqrt{\lambda}(\cos \sqrt{\lambda}-1)}$, I is the identity operator.

c) Convolution of functions $g$ and $f$ belong to the domain of operator $\mathcal{B}$ if $g \in D(\mathcal{B})$ and the identity

holds true.

$$
\mathcal{B}(g * f)=\mathcal{B} g * f
$$

d) The convolution generated by operator $\mathcal{B}$ has no annihilator, i.e., if $g * f \equiv 0$ is valid for each $g \in L_{2}(0,1)$, then $f \equiv 0$.

Доказательство. а) The bilinearity and associativity of introduced convolution is checked trivially. Let us show that the convolution is commutative in the space of summable functions. We introduce the integrals

$$
\begin{array}{ll}
I_{1}(g, f)=\int_{x}^{1} g(1+x-t) f(t) d t, & I_{2}(g, f)=\int_{0}^{x} g(x-t) f(t) d t \\
I_{3}(g, f)=\int_{0}^{1-x} g(1-x-t) f(t) d t, & I_{4}(g, f)=\int_{1-x}^{1} g(x-1+t) f(t) d t, \\
I_{5}(g, f)=\int_{0}^{x} g(1+t-x) f(t) d t, &
\end{array}
$$

where $x \in(0,1)$.

It is easy to make sure that

$$
I_{k}(g, f)=I_{k}(f, g) \quad \text { as } \quad k=1,2,3 .
$$

Let us consider $I_{4}(g, f)$ and let us make the change $\tau=x-1+t$ in this integral. We have

$$
I_{4}(g, f)=\int_{0}^{x} g(\tau) f(1-x+\tau) d \tau .
$$

It implies that $I_{4}(g, f)=I_{5}(f, g)$. Exactly in the same way we check the relation $I_{5}(g, f)=$ $I_{4}(f, g)$. Since by the definition of the convolution we have

$$
g * f=\frac{1}{2} I_{1}(g, f)+I_{2}(g, f)-\frac{1}{2} I_{3}(g, f)+\frac{1}{2} I_{4}(g, f)+\frac{1}{2} I_{5}(g, f),
$$

it implies the commutation identity $g * f=f * g$.

b) We denote

$$
y(x, \lambda)=(g * f)(x),
$$

where $g(x, \lambda)=\frac{\sin \sqrt{\lambda} x}{\sqrt{\lambda}(\cos \sqrt{\lambda}-1)}$. Let us calculate the derivative of $y(x, \lambda)$ w.r.t. $x$ :

$$
\begin{aligned}
y^{\prime}(x, \lambda)= & \frac{1}{\sqrt{\lambda}(\cos \sqrt{\lambda}-1)} \frac{d}{d x}\left[\frac{1}{2} \int_{x}^{1} \sin \sqrt{\lambda}(1+x-t) f(t) d t+\int_{0}^{x} \sin \sqrt{\lambda}(x-t) f(t) d t\right. \\
& -\frac{1}{2} \int_{0}^{1-x} \sin \sqrt{\lambda}(1-x-t) f(t) d t+\frac{1}{2} \int_{1-x}^{1} \sin \sqrt{\lambda}(x-1+t) f(t) d t \\
& \left.+\frac{1}{2} \int_{0}^{x} \sin \sqrt{\lambda}(1+t-x) f(t) d t\right] \\
= & \frac{f(x)}{2 \sqrt{\lambda}(\cos \sqrt{\lambda}-1)}[-\sin \sqrt{\lambda}+\sin \sqrt{\lambda}] \\
& +\frac{1}{\sqrt{\lambda}(\cos \sqrt{\lambda}-1)}\left[\frac{1}{2} \int_{x}^{1} \cos \sqrt{\lambda}(1-x-t) f(t) d t+\int_{0}^{x} \cos \sqrt{\lambda}(x-t) f(t) d t\right.
\end{aligned}
$$




$$
\begin{aligned}
& +\frac{1}{2} \int_{0}^{1-x} \cos \sqrt{\lambda}(1-x-t) f(t) d t+\frac{1}{2} \int_{1-x}^{1} \cos \sqrt{\lambda}(x-1+t) f(t) d t \\
& \left.-\frac{1}{2} \int_{0}^{x} \cos \sqrt{\lambda}(1+t-x) f(t) d t\right] .
\end{aligned}
$$

In the same way we calculate the second derivative of $y(x, \lambda)$ w.r.t. $x$. Finally we have

$$
y^{\prime \prime}(x, \lambda)=\frac{1}{2 \sqrt{\lambda}(\cos \sqrt{\lambda}-1)}[-\cos \sqrt{\lambda} f(x)+2 f(x)-f(x)-f(x)-\cos \sqrt{\lambda} f(x)]-\lambda y(x, \lambda) .
$$

It implies that $y(x, \lambda)$ satisfies the equation

$$
-y^{\prime \prime}(x, \lambda)=\lambda y(x, \lambda)+f(x) .
$$

It remains to check the boundary conditions. The first boundary condition is checked straightforwardly by substituting $x=0$ :

$$
y(0, \lambda)=\frac{1}{2 \sqrt{\lambda}(\cos \sqrt{\lambda}-1)}\left[\int_{0}^{1} \sin \sqrt{\lambda}(1-t) f(t) d t-\int_{0}^{1} \sin \sqrt{\lambda}(1-t) f(t) d t\right]=0 .
$$

We check the second boundary condition:

$$
\begin{aligned}
& y^{\prime}(0, \lambda)=\frac{1}{2 \sqrt{\lambda}(\cos \sqrt{\lambda}-1)}[\left.\int_{0}^{1} \cos \sqrt{\lambda}(1-t) f(t) d t+\int_{0}^{1} \cos \sqrt{\lambda}(1-t) f(t) d t\right], \\
& y^{\prime}(1, \lambda)=\frac{1}{2 \sqrt{\lambda}(\cos \sqrt{\lambda}-1)}\left[2 \int_{0}^{1} \cos \sqrt{\lambda}(1-t) f(t) d t\right. \\
&\left.+\int_{0}^{1} \cos (\sqrt{\lambda} t) f(t) d t-\int_{0}^{1} \cos (\sqrt{\lambda} t) f(t) d t\right] .
\end{aligned}
$$

It implies $y^{\prime}(0)=y^{\prime}(1)$.

c) Let $g \in D(\mathcal{B})$. We consider the convolution $g * f$, where $f \in L_{2}(0,1)$. Since $g \in D(\mathcal{B})$, then $g * f \in W_{2}^{2}[0,1]$. Indeed, the following representation of the first and the second derivatives of the convolution w.r.t. $x$ hold:

$$
\begin{aligned}
\frac{d}{d x}(g * f)(x)= & \int_{x}^{1} g^{\prime}(1+x-t) f(t) d t+\int_{1-x}^{1} g^{\prime}(x-1+t) f(t) d t+2 \int_{0}^{x} g^{\prime}(x-t) f(t) d t \\
& +\int_{0}^{1-x} g^{\prime}(1-x-t) f(t) d t-\int_{0}^{x} g^{\prime}(1+t-x) f(t) d t, \\
\frac{d^{2}}{d x^{2}}(g * f)(x)= & \left(g^{\prime \prime} * f\right)(x) .
\end{aligned}
$$

Here we have employed essentially that $g(0)=0$ and $g^{\prime}(0)=g^{\prime}(1)$.

d) Suppose that $g * f \equiv 0$ for each $g \in L_{2}(0,1)$. We introduce the function $g(x)=$ $\left.\frac{\sin \sqrt{\lambda} x}{\sqrt{\lambda}(\cos \sqrt{\lambda}-1)}\right|_{\lambda=1}$, which is well-defined since $\cos 1 \neq 1$. In accordance with Statement b), convolution $g * f$ means $(\mathcal{B}-I)^{-1} f$. If $(\mathcal{B}-I)^{-1} f(x) \equiv 0$, then $f(x) \equiv 0$. The proof is complete.

We provide one more useful identity.

Lemma 2.2. For each complex $\alpha$ and $\beta$ the identity

$$
\frac{\sin (\alpha x)}{\alpha} * \frac{\sin (\beta x)}{\beta}=\left(\frac{\sin (\alpha x)}{\alpha}(\cos \beta-1)-\frac{\sin (\beta x)}{\beta}(\cos \alpha-1)\right) /\left(\beta^{2}-\alpha^{2}\right)
$$

holds true. 
Доказательство. We denote

$$
y(x)=\frac{\sin (\alpha x)}{\alpha} * \frac{\sin (\beta x)}{\beta}
$$

and

$$
u(x)=\left(\frac{\sin (\alpha x)}{\alpha}(\cos \beta-1)-\frac{\sin (\beta x)}{\beta}(\cos \alpha-1)\right) /\left(\beta^{2}-\alpha^{2}\right) .
$$

It follows from Statement b) of Lemma 2.1 that function $y(x)$ satisfies the equation

$$
-y^{\prime \prime}(x)=\alpha^{2} y(x)+\frac{\sin (\beta x)}{\beta}(\cos \alpha-1)
$$

and the boundary conditions

$$
y(0)=0, y^{\prime}(0)=y^{\prime}(1) .
$$

Let us show that function $u(x)$ also satisfy equation (2.2) and boundary conditions (2.3) and it will complete the proof.

We find the first and the second derivative of function $u(x)$ :

$$
\begin{aligned}
u^{\prime}(x)= & \left(\alpha \frac{\cos (\alpha x)}{\alpha}(\cos \beta-1)-\beta \frac{\cos (\beta x)}{\beta}(\cos \alpha-1)\right) /\left(\beta^{2}-\alpha^{2}\right) \\
u^{\prime \prime}(x)= & \left(-\alpha^{2} \frac{\sin (\alpha x)}{\alpha}(\cos \beta-1)+\beta^{2} \frac{\sin (\beta x)}{\beta}(\cos \alpha-1)\right) /\left(\beta^{2}-\alpha^{2}\right) \\
= & -\alpha^{2}\left(\frac{\sin (\alpha x)}{\alpha}(\cos \beta-1)-\frac{\sin (\beta x)}{\beta}(\cos \alpha-1)\right) /\left(\beta^{2}-\alpha^{2}\right) \\
& +\left(\beta^{2}-\alpha^{2}\right)\left(\frac{\sin (\beta x)}{\beta}(\cos \alpha-1)\right) /\left(\beta^{2}-\alpha^{2}\right) \\
= & -\alpha^{2} u(x)+\frac{\sin (\beta x)}{\beta}(\cos \alpha-1) .
\end{aligned}
$$

Hence, function $u(x)$ satisfies equation 2.2 . Simple calculations

$$
\begin{aligned}
u(0) & =\left(\frac{\sin (\alpha 0)}{\alpha}(\cos \beta-1)-\frac{\sin (\beta 0)}{\beta}(\cos \alpha-1)\right) /\left(\beta^{2}-\alpha^{2}\right)=0, \\
u^{\prime}(0) & =\left(\alpha \frac{1}{\alpha}(\cos \beta-1)-\beta \frac{1}{\beta}(\cos \alpha-1)\right) /\left(\beta^{2}-\alpha^{2}\right) \\
& \left.=((\cos \beta-1)-(\cos \alpha-1)) /\left(\beta^{2}-\alpha^{2}\right)=(\cos \beta-\cos \alpha)\right) /\left(\beta^{2}-\alpha^{2}\right), \\
u^{\prime}(1) & =\left(\alpha \frac{\cos \alpha}{\alpha}(\cos \beta-1)-\beta \frac{\cos \beta}{\beta}(\cos \alpha-1)\right) /\left(\beta^{2}-\alpha^{2}\right) \\
& =(\cos \alpha(\cos \beta-1)-\cos \beta(\cos \alpha-1)) /\left(\beta^{2}-\alpha^{2}\right)=(\cos \beta-\cos \alpha) /\left(\beta^{2}-\alpha^{2}\right),
\end{aligned}
$$

lead us to boundary conditions 2.3 . The proof is complete.

We rewrite formula 2.1 as

$$
\sin (\alpha x) * \sin (\beta x)=\frac{(\beta \sin (\alpha x)(\cos \beta-1)-\alpha \sin (\beta x)(\cos \alpha-1))}{\beta^{2}-\alpha^{2}} .
$$

Lemma 2.3. For $\xi \in \mathbb{Z}_{+}$the identity

$$
\sin (2 \pi \xi x) * \sin (2 \pi \xi x)=0
$$

holds true. 
Доказательство. То prove the lemma, we make use of the following identities implied by formula (2.4) and based on simple substitutions and calculations. Substituting $\beta=2 \pi \xi$ for $\xi>0$ in formula 2.4 , we obtain that

$$
\sin (\alpha x) * \sin (2 \pi \xi x)=\frac{\alpha \sin (2 \pi \xi x)(\cos \alpha-1)}{\alpha^{2}-(2 \pi \xi)^{2}} .
$$

Passing to the limit as $\alpha \rightarrow 2 \pi \xi$ in $(2.5)$, we get

$$
\sin (2 \pi \xi x) * \sin (2 \pi \xi x)=\lim _{\alpha \rightarrow 2 \pi \xi} \sin (\alpha x) * \sin (2 \pi \xi x)=\lim _{\alpha \rightarrow 2 \pi \xi} \frac{\alpha \sin (2 \pi \xi x)(\cos \alpha-1)}{\alpha^{2}-(2 \pi \xi)^{2}}=0 .
$$

The proof is complete.

We observe that for each integer $\eta$

$$
\sin (2 \pi \eta x) * \sin (2 \pi \xi x)=0,
$$

since $\cos \alpha-1=0$ as $\alpha=2 \pi \xi$.

Lemma 2.4. For $\xi \in \mathbb{Z}_{+}$the identity

$$
x \cos (2 \pi \xi x) * \sin (2 \pi \xi x)=-\frac{1}{4} \sin (2 \pi \xi x)
$$

holds true.

Доказательство. We differentiate identity (2.5) w.r.t. $\alpha$

$$
x \cos (\alpha x) * \sin (2 \pi \xi x)=\sin (2 \pi \xi x) \frac{[\cos \alpha-1-\alpha \sin \alpha]\left(\alpha^{2}-(2 \pi \xi)^{2}\right)-2 \alpha^{2}(\cos \alpha-1)}{\left(\alpha^{2}-(2 \pi \xi)^{2}\right)^{2}} .
$$

Passing to the limit as $\alpha \rightarrow 2 \pi \xi$, by $(2.9)$ we have

$$
\begin{aligned}
x \cos (2 \pi \xi x) * \sin (2 \pi \xi x) & =\lim _{\alpha \rightarrow 2 \pi \xi} x \cos (\alpha x) * \sin (2 \pi \xi x) \\
& =\sin (2 \pi \xi x) \lim _{\alpha \rightarrow 2 \pi \xi} \frac{[\cos \alpha-1-\alpha \sin \alpha]\left(\alpha^{2}-(2 \pi \xi)^{2}\right)-2 \alpha^{2}(\cos \alpha-1)}{\left(\alpha^{2}-(2 \pi \xi)^{2}\right)^{2}} \\
& =-\sin (2 \pi \xi x)\left[\lim _{\alpha \rightarrow 2 \pi \xi} \frac{\alpha \sin \alpha}{\alpha^{2}-(2 \pi \xi)^{2}}+2 \lim _{\alpha \rightarrow 2 \pi \xi} \frac{\alpha^{2}(\cos \alpha-1)}{\left(\alpha^{2}-(2 \pi \xi)^{2}\right)^{2}}\right] \\
& =-\sin (2 \pi \xi x)\left[\frac{1}{2}-\frac{1}{4}\right]=-\frac{1}{4} \sin (2 \pi \xi x) .
\end{aligned}
$$

Thus, identity (2.8) holds true. The proof is complete.

Lemma 2.5. For $\xi \in \mathbb{Z}_{+}$the identity

$$
x \cos (2 \pi \xi x) * x \cos (2 \pi \xi x)=-\frac{1}{4} \sin (2 \pi \xi x)-\frac{1}{4} x \cos (2 \pi \xi x)
$$

holds true.

Доказательство. We calculate

$$
\begin{aligned}
& x \cos (2 \pi \xi x) * x \cos (2 \pi \xi x)=\lim _{\alpha \rightarrow 2 \pi \xi} \frac{d}{d \alpha}\left[\lim _{\beta \rightarrow 2 \pi \xi} \frac{d}{d \beta}(\sin (\alpha x) * \sin (\beta x))\right] \\
& =\lim _{\alpha \rightarrow 2 \pi \xi} \frac{d}{d \alpha}\left[\lim _{\beta \rightarrow 2 \pi \xi} \frac{d}{d \beta}\left(\frac{(\beta \sin (\alpha x)(\cos \beta-1)-\alpha \sin (\beta x)(\cos \alpha-1))}{\beta^{2}-\alpha^{2}}\right)\right] \\
& =\lim _{\alpha \rightarrow 2 \pi \xi} \frac{d}{d \alpha}\left[\lim _{\beta \rightarrow 2 \pi \xi} K(\alpha, \beta)\right],
\end{aligned}
$$


where

$$
\begin{aligned}
K(\alpha, \beta)= & \frac{\sin (\alpha x)(\cos \beta-1-\beta \sin \beta)-\alpha x \cos (\beta x)(\cos \alpha-1)}{\beta^{2}-\alpha^{2}} \\
& -\frac{2 \beta(\beta \sin (\alpha x)(\cos \beta-1)-\alpha \sin (\beta x)(\cos \alpha-1))}{\left(\beta^{2}-\alpha^{2}\right)^{2}}
\end{aligned}
$$

Since

$$
\lim _{\beta \rightarrow 2 \pi \xi} K(\alpha, \beta)=-x \cos (2 \pi \xi x) \frac{\alpha(\cos \alpha-1)}{(2 \pi \xi)^{2}-\alpha^{2}}+4 \pi \xi \sin (2 \pi \xi x) \frac{\alpha(\cos \alpha-1)}{\left((2 \pi \xi)^{2}-\alpha^{2}\right)^{2}},
$$

we arrive at

$$
\begin{aligned}
& x \cos (2 \pi \xi x) * x \cos (2 \pi \xi x) \\
& \quad=\lim _{\alpha \rightarrow 2 \pi \xi} \frac{d}{d \alpha}\left[-x \cos (2 \pi \xi x) \frac{\alpha(\cos \alpha-1)}{(2 \pi \xi)^{2}-\alpha^{2}}+4 \pi \xi \sin (2 \pi \xi x) \frac{\alpha(\cos \alpha-1)}{\left((2 \pi \xi)^{2}-\alpha^{2}\right)^{2}}\right]=\lim _{\alpha \rightarrow 2 \pi \xi} F(\alpha),
\end{aligned}
$$

where

$$
\begin{aligned}
F(\alpha)= & -x \cos (2 \pi \xi x)\left(\frac{\cos \alpha-1-\alpha \sin \alpha}{(2 \pi \xi)^{2}-\alpha^{2}}+2 \alpha \frac{\alpha(\cos \alpha-1)}{\left((2 \pi \xi)^{2}-\alpha^{2}\right)^{2}}\right) \\
& +4 \pi \xi \sin (2 \pi \xi x)\left(\frac{\cos \alpha-1-\alpha \sin \alpha}{\left((2 \pi \xi)^{2}-\alpha^{2}\right)^{2}}+4 \alpha \frac{\alpha(\cos \alpha-1)}{\left((2 \pi \xi)^{2}-\alpha^{2}\right)^{3}}\right) .
\end{aligned}
$$

We calculate

$$
\lim _{\alpha \rightarrow 2 \pi \xi} F(\alpha)=-\frac{1}{4} \sin (2 \pi \xi x)-\frac{1}{4} x \cos (2 \pi \xi x) .
$$

We finally have

$$
x \cos (2 \pi \xi x) * x \cos (2 \pi \xi x)=-\frac{1}{4} \sin (2 \pi \xi x)-\frac{1}{4} x \cos (2 \pi \xi x) .
$$

The proof is complete.

The next identity is checked by straightforward calculations

$$
x * x=\frac{1}{2} x .
$$

In the same way it is easy to show that for $\xi \neq \eta>0$ the identities

$$
\begin{aligned}
& \sin (2 \pi \xi x) * \sin (2 \pi \eta x)=0, x \cos (2 \pi \xi x) * \sin (2 \pi \eta x)=0, \\
& x \cos (2 \pi \xi x) * x \cos (2 \pi \eta x)=0, \sin (2 \pi \xi x) * x=0, x \cos (2 \pi \xi x) * x=0 .
\end{aligned}
$$

hold true.

\section{Test functions, $\mathcal{B}$-Fourier transform and Sobolev spaces $\mathcal{B} W_{2}^{s}[0,1]$}

While introducing distributions on $\mathbb{R}$, an important role is played by a class of test functions. As test functions on $\mathbb{R}$, infinitely differentiable compactly supported functions can serve. In studying periodic processes, infinitely differentiable periodic functions are employed as test functions. In our case, as test functions, we consider the class $D\left(\mathcal{B}^{\infty}\right)=\bigcap_{m=1}^{\infty} D\left(\mathcal{B}^{m}\right)$, where $D\left(\mathcal{B}^{m}\right)$ is the domain of operator $\mathcal{B}^{m}$ for $m \geqslant 1$.

In the next lemma we solve the issue on existence of test functions in $D\left(\mathcal{B}^{\infty}\right)$.

Lemma 3.1. If $g \in D\left(\mathcal{B}^{\infty}\right)$, then for each $f \in L_{2}(0,1)$ we have $g * f \in D\left(\mathcal{B}^{\infty}\right)$. 
The statement of this lemma is implied by Statement c) of Lemma 2.1.

In accordance with Lemma 3.1, it is sufficient to construct one function $g \in D\left(\mathcal{B}^{\infty}\right)$ to obtain infinitely many other functions in $D\left(\mathcal{B}^{\infty}\right)$. As $g$ we can take eigenfunctions and adjoint functions of operator $\mathcal{B}$. We need to choose probably a finite set of functions $g_{1}, g_{2}, \ldots$ in $D\left(\mathcal{B}^{\infty}\right)$ so that the closure of linear span $\operatorname{span}\left\{g_{s} * f: s=1,2, \ldots, f \in L_{2}(0,1)\right\}$ coincides with $D\left(\mathcal{B}^{\infty}\right)$. In order to define the closure, we need to introduce a topology in linear space $D\left(\mathcal{B}^{\infty}\right)$. We shall say that a sequence of functions $y_{j}$ in $D\left(\mathcal{B}^{\infty}\right)$ converges to zero as $j \rightarrow \infty$ in the sense of topology in $D\left(\mathcal{B}^{\infty}\right)$ if for each $m=1,2, \ldots$

$$
\mathcal{B}^{m} y_{j} \rightrightarrows^{[0,1]} 0, j \rightarrow \infty
$$

where $\rightrightarrows^{[0,1]}$ denotes the uniform in $[0,1]$ convergence.

We introduce the notation $C_{\mathcal{B}}^{\infty}[0,1]:=D\left(\mathcal{B}^{\infty}\right)$. In fact, functions in $C_{\mathcal{B}}^{\infty}[0,1]$ are defined only on the interval $[0,1]$. In what follows, we extend functions $C_{\mathcal{B}}^{\infty}[0,1]$ continuously on the whole axis.

We begin with continuing functions in $C_{\mathcal{B}}^{\infty}[0,1]$ into $[-1,0)$. In view of the boundary condition $y(0)=0$, we continue this function by the oddness, i.e.,

$$
y(0-x)=-y(0+x), \quad x \in(0,1]
$$

or

$$
y(x)=-y(-x), \quad x \in[-1,0) .
$$

Now we define function $y$ inductively on $I_{n}=(n, n+1]$ as follows

$$
y(x)=2 y(x-1)-y(x-2), \quad x \in I_{n} .
$$

Definition 3.1. The space $\mathcal{D}_{\mathcal{B}}^{\prime}(0,1):=\mathcal{L}\left(C_{\mathcal{B}}^{\infty}[0,1], \mathbb{C}\right)$ dual to $C_{\mathcal{B}}^{\infty}[0,1]$ is called a space of $\mathcal{B}$-distributions. For $u \in \mathcal{D}_{\mathcal{B}}^{\prime}(0,1) u \varphi \in C_{\mathcal{B}}^{\infty}[0,1]$ we write

$$
u(\varphi)=\langle u, \varphi\rangle \text {. }
$$

For each $\psi \in C_{\mathcal{B}}^{\infty}[0,1]$ the mapping

$$
\varphi \mapsto \int_{0}^{1} \varphi(x) \overline{\psi(x)} d x
$$

is a $\mathcal{B}$-distribution that implies $\psi \in C_{\mathcal{B}}^{\infty}[0,1] \subset \mathcal{D}_{\mathcal{B}}^{\prime}(0,1)$.

Definition 3.2. By $\mathcal{S}\left(\mathbb{Z}_{+}\right)$we denote the space of rapidly decaying functions acting from $\mathbb{Z}_{+}$into $\mathbb{C}$. That is, $\varphi \in \mathcal{S}\left(\mathbb{Z}_{+}\right)$if for an arbitrary $M<\infty$ there exists a constant $C_{\varphi, M}$ such that the estimate

$$
|\varphi(\xi)| \leqslant C_{\varphi, M}\langle\xi\rangle^{-M}
$$

holds true for all $\xi \in \mathbb{Z}_{+}$, where

$$
\langle\xi\rangle:=\left(1+\sqrt{\lambda_{\xi}}\right) .
$$

The topology on $\mathcal{S}\left(\mathbb{Z}_{+}\right)$is defined by semi-norms $p_{k}$, where $k \in \mathbb{Z}_{+}$and $p_{k}(\varphi):=$ $\sup _{\xi \in \mathbb{Z}_{+}}\langle\xi\rangle^{k}|\varphi(\xi)|$.

Remark 3.1. Linear continuous functionals on $\mathcal{S}\left(\mathbb{Z}_{+}\right)$read as

$$
\varphi \mapsto\langle u, \varphi\rangle:=\sum_{\xi \in \mathbb{Z}} u(\xi) \varphi(\xi),
$$

where functions $u: \mathbb{Z}_{+} \rightarrow \mathbb{C}$ grow polynomially at infinity, i.e., there exist constants $M<\infty$ and $C_{u, M}$ such that the inequality

$$
|u(\xi)| \leqslant C_{u, M}\langle\xi\rangle^{M}
$$

holds true for all $\xi \in \mathbb{Z}_{+}$. Such distributions $u: \mathbb{Z}_{+} \rightarrow \mathbb{C}$ form space $\mathcal{S}^{\prime}\left(\mathbb{Z}_{+}\right)$. 
Definition 3.3. Given $f \in C_{\mathcal{B}}^{\infty}[0,1]$, we introduce $\mathcal{B}$-Fourier transform by the formula

$$
\widehat{f}(\xi):=\left(\mathcal{F}_{\mathcal{B}} f\right)(\xi)=\int_{0}^{1} f(x) v_{\xi}(x) d x .
$$

In the same way, for $f \in C_{\mathcal{B}^{*}}^{\infty}[0,1]$ we introduce $\mathcal{B}^{*}$-Fourier transform by the formula

$$
\widehat{f}^{*}(\xi):=\left(\mathcal{F}_{\mathcal{B}^{*}} f\right)(\xi)=\int_{0}^{1} f(x) u_{\xi}(x) d x .
$$

Lemma 3.2. $\mathcal{F}_{\mathcal{B}}$ is a continuous bijection

$$
\left(\mathcal{F}_{\mathcal{B}} f\right)(\xi)=(f \mapsto \widehat{f}(\xi)): C_{\mathcal{B}}^{\infty}[0,1] \rightarrow \mathcal{S}\left(\mathbb{Z}_{+}\right),
$$

and the inverse transform $\mathcal{F}_{\mathcal{B}}^{-1}: \mathcal{S}\left(\mathbb{Z}_{+}\right) \rightarrow C_{\mathcal{B}}^{\infty}[0,1]$ is defined by the formula

$$
f(x)=\sum_{\xi \in \mathbb{Z}_{+}} \widehat{f}(\xi) u_{\xi}(x) .
$$

Similarly, $\mathcal{F}_{\mathcal{B}^{*}}$ is a continuous bijection

$$
\left(\mathcal{F}_{\mathcal{B}^{*}} f\right)(\xi)=\left(f \mapsto \widehat{f}^{*}(\xi)\right): C_{\mathcal{B}^{*}}^{\infty}[0,1] \rightarrow \mathcal{S}\left(\mathbb{Z}_{+}\right)
$$

and the inverse transform $\mathcal{F}_{\mathcal{B}^{*}}^{-1}: \mathcal{S}\left(\mathbb{Z}_{+}\right) \rightarrow C_{\mathcal{B}^{*}}^{\infty}[0,1]$ is defined by the formula

$$
f(x)=\sum_{\xi \in \mathbb{Z}_{+}} \widehat{f}^{*}(\xi) v_{\xi}(x) .
$$

Доказательство. The proof of Lemma 3.2 is in fact the same as for the classical periodic case except the steps, where biorthogonality properties are essentially employed. Let us show first that for $f \in C_{\mathcal{B}}^{\infty}[0,1]$, we have $\widehat{f} \in \mathcal{S}\left(\mathbb{Z}_{+}\right)$, i.e., for each $M<\infty$ there exists a constant $C$ such that the estimate

$$
|\widehat{f}(\xi)| \leqslant C\langle\xi\rangle^{-M}
$$

holds true for all $\xi \in \mathbb{Z}_{+}$. Indeed, for an arbitrary $M \in \mathbb{N}$ and even $\xi$ we obtain the inequality

$$
\begin{aligned}
|\widehat{f}(\xi)| & =\left|\int_{0}^{1} f(x) v_{\xi}(x) d x\right|=\left|\int_{0}^{1} f(x) \frac{\left(\mathcal{B}^{*}\right)^{M} v_{\xi}(x)}{\lambda_{\xi}^{M}} d x\right|= \\
& =\left|\frac{1}{\lambda_{\xi}^{M}} \int_{0}^{1} \mathcal{B}^{M} f(x) v_{\xi}(x) d x\right| \leqslant C\left\|\mathcal{B}^{M} f\right\|_{L_{2}(0,1)}\langle\xi\rangle^{-2 M} .
\end{aligned}
$$

The same estimate is true for odd $\xi$. Thus, for $f \in C_{\mathcal{B}}^{\infty}[0,1]$ we have $\widehat{f} \in \mathcal{S}\left(\mathbb{Z}_{+}\right)$. Since $\left\|\mathcal{B}^{M} f\right\|_{L_{2}(0,1)}$ define semi-norms in space $f C_{\mathcal{B}}^{\infty}[0,1]$, it implies the continuity of operator $\mathcal{F}_{\mathcal{B}}$ from $C_{\mathcal{B}}^{\infty}[0,1]$ into $\mathcal{S}\left(\mathbb{Z}_{+}\right)$.

It is clear that for $h \in \mathcal{S}\left(\mathbb{Z}_{+}\right)$formula 3.3 defines a function $\mathcal{F}_{\mathcal{B}}^{-1} h \in C_{\mathcal{B}}^{\infty}[0,1]$ with Fourier coefficient $h(\xi)$. If two functions $f_{1}, f_{2} \in C_{\mathcal{B}}^{\infty}[0,1]$ have the same Fourier coefficients $\widehat{f}_{1}(\xi)=$ $\widehat{f}_{2}(\xi) \xi \in \mathbb{Z}_{+}$, by the density of the linear span $\left\{u_{\xi}\right\}_{\xi \in \mathbb{Z}_{+}}$in $C_{\mathcal{B}}^{\infty}[0,1]$, we obtain

$$
f_{1}(x)=\sum_{\xi \in \mathbb{Z}_{+}} \widehat{f}_{1}(\xi) u_{\xi}(x)=\sum_{\xi \in \mathbb{Z}_{+}} \widehat{f}_{2}(\xi) u_{\xi}(x)=f_{2}(x) .
$$

The continuity of the mapping $\mathcal{F}_{\mathcal{B}}^{-1}: \mathcal{S}\left(\mathbb{Z}_{+}\right) \rightarrow C_{\mathcal{B}}^{\infty}[0,1]$ is proved by the same arguments. The properties of the adjoint transform $\mathcal{F}_{\mathcal{B}^{*}}$ are proved in the same way. The proof is complete.

By means of the inverse $\mathcal{B}$-Fourier transform $\mathcal{F}_{\mathcal{B}}^{-1}: \mathcal{S}\left(\mathbb{Z}_{+}\right) \rightarrow C_{\mathcal{B}}^{\infty}[0,1]$ we uniquely continue $\mathcal{B}$-Fourier transform to the mapping

$$
\mathcal{F}_{\mathcal{B}}: \mathcal{D}_{\mathcal{B}}^{\prime}(0,1) \rightarrow \mathcal{S}^{\prime}\left(\mathbb{Z}_{+}\right)
$$


by the formula

$$
\left\langle\mathcal{F}_{\mathcal{B}} w, \varphi\right\rangle:=\left\langle w, \overline{\mathcal{F}_{\mathcal{B}^{*}}^{-1} \bar{\varphi}}\right\rangle, \quad \text { для } w \in \mathcal{D}_{\mathcal{B}}^{\prime}(0,1), \varphi \in \mathcal{S}\left(\mathbb{Z}_{+}\right) .
$$

It follows that if $w \in \mathcal{D}_{\mathcal{B}}^{\prime}(0,1)$, then $\widehat{w} \in \mathcal{S}^{\prime}\left(\mathbb{Z}_{+}\right)$. We also observe that if $w \in C_{\mathcal{B}}^{\infty}[0,1]$, then the identity

$$
\begin{aligned}
\langle\widehat{w}, \varphi\rangle & =\sum_{\xi \in \mathbb{Z}_{+}} \widehat{w}(\xi) \varphi(\xi)=\sum_{\xi \in \mathbb{Z}_{+}}\left(\int_{0}^{1} w(x) \overline{v_{\xi}(x)} d x\right) \varphi(\xi) \\
& =\int_{0}^{1} w(x) \overline{\left(\sum_{\xi \in \mathbb{Z}_{+}} \overline{\varphi(\xi)} v_{\xi}(x)\right)} d x=\int_{0}^{1} w(x) \overline{\left(\mathcal{F}_{\mathcal{B}^{*}}^{-1} \bar{\varphi}\right)} d x=\left\langle w, \overline{\mathcal{F}_{\mathcal{B}^{*}}^{-1} \bar{\varphi}}\right\rangle
\end{aligned}
$$

holds true.

In the same way we define the mapping

$$
\mathcal{F}_{\mathcal{B}^{*}}: \mathcal{D}_{\mathcal{B}^{*}}^{\prime}(0,1) \rightarrow \mathcal{S}^{\prime}\left(\mathbb{Z}_{+}\right)
$$

In the space of sequences $X=\prod_{k=0}^{\infty} \mathbb{C}^{m_{k}}$ we introduce the inner Cauchy convolution, where indices $m_{k}$ in Lemma 1.1 are $m_{0}=1$ and $m_{i}=2, i \geqslant 1$. Let $\xi=\left\{\xi_{0} ; \xi_{2 k-1}, \xi_{2 k}, k>0\right\}$ and $\eta=\left\{\eta_{0} ; \eta_{2 k-1}, \eta_{2 k}, k>0\right\}$ are the elements of $X=\prod_{k=0}^{\infty} \mathbb{C}^{m_{k}}$. Its convolution is the sequence $\theta=\left\{\theta_{0} ; \theta_{2 k-1}, \theta_{2 k}, k>0\right\}$, where

$$
\theta_{0}=\xi_{0} \eta_{0}, \quad \theta_{2 k-1}=\xi_{2 k-1} \eta_{2 k}+\xi_{2 k} \eta_{2 k}+\xi_{2 k} \eta_{2 k-1}, \quad \theta_{2 k}=\xi_{2 k} \eta_{2 k} .
$$

The obtained in this way convolution will be denoted by $\xi *_{X} \eta:=\theta$. The introduced convolutions $*_{X}$ and $*$ are related by the Fourier transform.

Theorem 3.1. For each two elements $f, g \in C_{\mathcal{B}}^{\infty}[0,1]$ the identity

$$
\widehat{f * g}=\widehat{f} *_{X} \widehat{g}
$$

holds true.

Доказательство. Taking into consideration identities (2.4)-(2.12) and Lemmata 2.3 2.5, we rewrite the convolution as follows

$$
\begin{aligned}
&(f * g)(x)= \sum_{\xi=0}^{\infty} \sum_{k=0}^{m_{\xi}} \widehat{f}(2 \xi-k) u_{2 \xi-k}(x) * \sum_{\eta=0}^{\infty} \sum_{s=0}^{m_{\eta}} \widehat{g}(2 \eta-s) u_{2 \eta-s}(x) \\
&=\frac{1}{2} \widehat{f}(0) \widehat{g}(0) u_{0}(x)-\frac{1}{4} \sum_{\xi=1}^{\infty}[(\widehat{f}(2 \xi-1) \widehat{g}(2 \xi)+\widehat{f}(2 \xi) \widehat{g}(2 \xi) \\
&\left.+\widehat{f}(2 \xi) \widehat{g}(2 \xi-1)) u_{2 \xi-1}(x)+\widehat{f}(2 \xi) \widehat{g}(2 \xi) u_{2 \xi}(x)\right] .
\end{aligned}
$$

Applying Fourier transform, we obtain the required identity. The proof is complete.

We introduce a space of sequences $\mathcal{B} l_{2}$ generated by the systems of functions $\left\{u_{\xi}\right\}_{\xi=0}^{\infty}$ and $\left\{v_{\xi}\right\}_{\xi=0}^{\infty}$ with the scalar product

$$
(\widehat{f}, \widehat{g})_{\mathcal{B} l_{2}}:=\sum_{\xi \in \mathbb{Z}_{+}} \widehat{f}(\xi) \overline{\widehat{g}^{*}(\xi)} .
$$

It is easy to make sure that space $\mathcal{B} l_{2}$ is a Hilbert one. Indeed,

$$
(\widehat{f}, \widehat{g})_{\mathcal{B} l_{2}}=\left(\widehat{f}, \widehat{g}^{*}\right)_{l_{2}} \text {. }
$$


Moreover,

$$
\begin{aligned}
(\widehat{f}, \widehat{g})_{\mathcal{B l}_{2}} & =\sum_{\xi \in \mathbb{Z}_{+}} \widehat{f}(\xi) \overline{\widehat{g}^{*}(\xi)}=\widehat{f}(0) \overline{\widehat{g}^{*}(0)}+\sum_{\xi \in \mathbb{N}} \sum_{i=1}^{2} \widehat{f}(2(\xi-1)+i) \overline{\widehat{g}^{*}(2(\xi-1)+i)} \\
& =\widehat{f}(0) \overline{\widehat{g}^{*}(0)}+\sum_{\xi \in \mathbb{N}} \sum_{\eta \in \mathbb{N}} \sum_{i=1}^{2} \widehat{f}(2(\xi-1)+i) \overline{\widehat{g}^{*}(2(\eta-1)+i)}\left(u_{2(\xi-1)+i}, v_{2(\eta-1)+i}\right)_{L_{2}} \\
& =(f, g)_{L_{2}} .
\end{aligned}
$$

Thus,

$$
(\widehat{f}, \widehat{g})_{\mathcal{B} l_{2}}=(f, g)_{L_{2}}
$$

The Hilbert space axioms are implied by the obtained identity. Hence, the Plancherel identity holds true.

Lemma 3.3 (Plancherel identity). If $f \in L_{2}(0,1)$, then $\widehat{f} \in \mathcal{B l}_{2}$ and

$$
\|f\|_{L_{2}}=\|\widehat{f}\|_{\mathcal{B} l_{2}} \text {. }
$$

Доказательство. Ву straightforward calculations we check that

$$
\begin{aligned}
\|f\|_{L_{2}}^{2} & =(f, f)_{L_{2}}=\left(\sum_{\xi \in \mathbb{Z}_{+}} \widehat{f}(\xi) u_{\xi}, \sum_{\eta \in \mathbb{Z}_{+}} \widehat{f}^{*}(\eta) v_{\eta}\right)_{L_{2}} \\
& =\widehat{f}(0) \overline{\widehat{g}^{*}(0)}+\sum_{\xi \in \mathbb{N}} \sum_{\eta \in \mathbb{N}} \sum_{i=1}^{2} \widehat{f}(2(\xi-1)+i) \overline{\widehat{g}^{*}(2(\eta-1)+i)}\left(u_{2(\xi-1)+i}, v_{2(\eta-1)+i}\right)_{L_{2}} \\
& =\sum_{\xi \in \mathbb{Z}_{+}} \widehat{f}(\xi) \overline{\widehat{g}^{*}(\xi)}=(\widehat{f}, \widehat{f})_{\mathcal{B} l_{2}}=\|\widehat{f}\|_{\mathcal{B} l_{2}}^{2} .
\end{aligned}
$$

It completes the proof.

In the next definition we introduce a Sobolev space generated by operator $\mathcal{B}$ :

Definition 3.4. (Sobolev space $\mathcal{B} W^{s}[0,1]$ ) Given $f \in \mathcal{D}_{\mathcal{B}}^{\prime}(0,1)$ and a number $s \in \mathbb{R}$, we define norm $\|\cdot\|_{\mathcal{B} W^{s}[0,1]}$ by the formula

$$
\|f\|_{\mathcal{B} W^{s}[0,1]}:=\left(\sum_{\xi \in \mathbb{Z}}\langle\xi\rangle^{2 s} \widehat{f}(\xi) \overline{\widehat{f}^{*}(\xi)}\right)^{1 / 2} .
$$

Sobolev space $\mathcal{B} W^{s}[0,1]$ is the space of $\mathcal{B}$-distributions $f$ for which $\|f\|_{\mathcal{B} W^{s}[0,1]}<\infty$. Or, for $f \in L_{2}(0,1): f \in \mathcal{B} W^{s}[0,1]$ if and only if $\langle\xi\rangle^{s} \widehat{f}(\xi) \in \mathcal{B} l_{2}$.

Lemma 3.4. For each $s \in \mathbb{R}$, Sobolev space $\mathcal{B} W^{s}[0,1]$ is a Hilbert space with the scalar product

$$
(f, g)_{\mathcal{B} W^{s}}:=\sum_{\xi \in \mathbb{Z}_{+}}\langle\xi\rangle^{2 s} \widehat{f}(\xi) \overline{\widehat{g}^{*}(\xi)} .
$$

Доказательство. Spaces $\mathcal{B} W^{0}[0,1]$ and $\mathcal{B} W^{s}[0,1]$ are isometrically isomorphic via the canonical isomorphism $\varphi_{s}: \mathcal{B} W^{0}[0,1] \rightarrow \mathcal{B} W^{s}[0,1]$ defined by the formula

$$
\varphi_{s} f(x):=\sum_{\xi \in \mathbb{Z}_{+}}\langle\xi\rangle^{-s} \widehat{f}(\xi) u_{\xi}(x) .
$$

Indeed, $\varphi_{s}$ is a linear isometry between $\mathcal{B} W^{t}[0,1]$ and $\mathcal{B} W^{t+s}[0,1]$ for each $s \in \mathbb{R}$ and the formulae $\varphi_{s_{1}} \varphi_{s_{2}}=\varphi_{s_{1}+s_{2}}$ and $\varphi_{s}^{-1}=\varphi_{-s}$ hold true. Thus, the density of space $L_{2}(0,1)=$ $\mathcal{B} W^{0}[0,1]$ is transferred for space $\mathcal{B} W^{s}[0,1]$ for each $s \in \mathbb{R}$. 


\section{BIBLIOGRAPHY}

1. B.E. Kanguzhin, S.N. Gani. Convolutions generated by differential operators on a segment // Izv. NAN RK. Ser. Fiz.-Mat. 1, 29-33 (2004). (in Russian).

2. B.E. Kanguzhin, D.B. Nurakhmetov. Nonlocal interior boundary value problems for differential operators and some related constructions // Math. J. 12:3, 92-100 (2012). (in Russian).

3. B. Kanguzhin and N. Tokmagambetov. The Fourier transform and convolutions generated by a differential operator with boundary condition on a segment // in book "Fourier Analysis: Trends in Mathematics". Birkhäuser, Basel, 235-251 (2014).

4. B. Kanguzhin, N. Tokmagambetov, K. Tulenov. Pseudo-differential operators generated by a nonlocal boundary value problem // Complex Var. Elliptic Equ. 60:1, 107-117 (2015).

5. M. Ruzhansky, N. Tokmagambetov. Nonharmonic analysis of boundary value problems // Int. Math. Res. Notices. (2015), to appear.

6. N.I. Ionkin. Solution of a boundary-value problem in heat conduction with a nonclassical boundary condition // Differ. Uravn. 13:2, 294-304 (1977). [Diff. Equat. 13, 204-211 (1977).]

7. A.M. Sedletskii. Biorthogonal expansions of functions in series of exponents on intervals of the real axis // Uspekhi Matem. Nauk. 37:5, 51-95 (1982). [Russ. Math. Surv. 37:5, 57-108 (1982).]

8. A.M. Sedletskii. Nonharmonic analysis // Itogi Nauki i Tekhniki. Ser. Sovrem. Mat. Pril. 96, 106-211 (2006). [J. Math. Sci. 116:5, 3551-3619 (2003).]

Baltabek Esmatovich Kanguzhin,

Al-Farabi Kazakh National University,

Al-Farabi av., 71,

050040, Almaty, Kazakhstan

E-mail: kanbalta@mail.ru

Niyaz Esenzholovich Tokmagambetov, Al-Farabi Kazakh National University, Al-Farabi av., 71, 050040, Almaty, Kazakhstan

E-mail: niyaz.tokmagambetov@gmail.com 